\title{
Camillo Benso Ballabio (1912 - 1984)
}

\author{
F. Fantini \\ Cattedra di Reumatologia, Università di Milano, Unità Operativa di Reumatologia, A.O. Istituto Ortopedico Gaetano Pini, Milano
}

\begin{abstract}
SUMMARY
The author commemorates, in the 20th year since his death, Camillo Benso Ballabio, unforgettable figure of rheumatologist, renowned clinician, man of great culture. The most important stages of his life are described, by adding to the biographical sketches some quotations that confirm the intellectual depth of such a protagonist of the history of the Italian rheumatology.
\end{abstract}

Reumatismo, 2004; 56(4):286-292

Commemorazione pronunciata

il giorno 23 giugno 2004,

in occasione dell'inaugurazione

delle XI Giornate Ortopedico-Reumatologiche

dell'Istituto Ortopedico Gaetano Pini

B ensogen enso Balabio (con una sola lettera 1 , come si legge nell'atto di nascita), nacque il 17 aprile 1912 da famiglia milanese. La famiglia era numerosa, il padre, un "viaggiatore di commercio" severo e autoritario, ambiva che tutti, comprese le figliole, studiassero e si facessero una posizione nel mondo del lavoro. Questo compito non risultò gravoso al giovanetto Benso che, dotato di ingegno vivace, intelligenza pronta e memoria eccezionale, non ebbe difficoltà alcuna a completare gli studi primari e secondari.

Nel 1930, i lusinghieri risultati conseguiti nell'esame di licenza liceale gli consentirono di vincere il concorso per l'ammissione al prestigioso Collegio Ghislieri di Pavia, permettendogli di iscriversi alla rinomata Facoltà di Medicina e Chirurgia dell'Alma Universitas Ticinensis. Al terzo anno di Medicina per il brillante studente pavese un decisivo avvenimento, che avrebbe condizionato il suo

Indirizzo per la corrispondenza:

Prof. Flavio Fantini

Unità Operativa di Reumatologia

A.O. Istituto Ortopedico Gaetano Pini

Piazza C. Ferrari, 1 - 20122 Milano

E-mail flavio.fantini@unimi.it futuro: è accolto in qualità di allievo interno nell'Istituto di Patologia Medica diretto dal Prof. Luigi Villa, che sarà il suo Maestro di scienza medica e la sua sicura guida nello studio e nella ricerca. Laureatosi nel luglio 1936 conseguendo voti assoluti e lode con una tesi sperimentale sul "complesso aurobatterico tubercolare", il giovane "dottorino", mentre iniziava con immediato successo la sua attività professionale, muoveva i primi passi nella carriera universitaria. Nel 1938 il Prof. Villa veniva chiamato ad occupare la Catte-

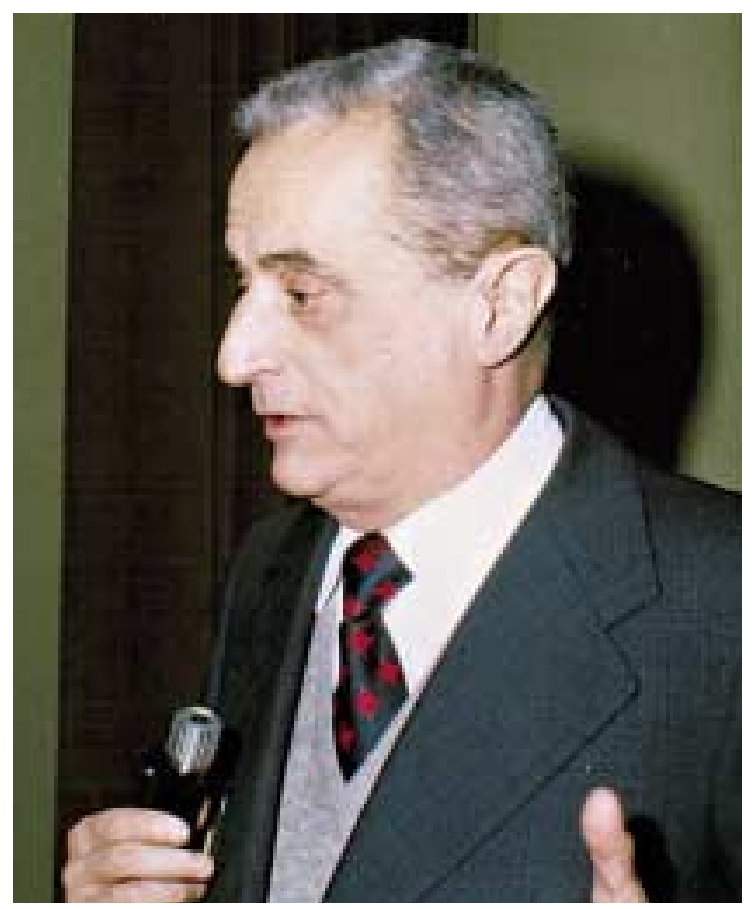


dra di Patologia Medica a Milano; qui lo seguiva il Balabio in qualità di "assistente volontario". Seguirono i tempi difficili della guerra: "assistente universitario incaricato" nell' anno accademico '42'43, da tale carica veniva sospeso in seguito alla riduzione di organico "per le esigenze belliche".

Dal 1945 Balabio, dietro consiglio del suo Maestro Villa, indirizzava i suoi studi verso la reumatologia e da quell'epoca, tra i primi in Italia, diveniva reumatologo "a tempo pieno". Nel 1951 conseguiva la Libera Docenza in Patologia Speciale Medica e Metodologia Clinica. Nel 1952 presso l'Istituto di Clinica Medica, dove dal 1949 il Prof. Villa e la sua Scuola si erano trasferiti, veniva istituito un Centro Cardio-Reumatologico, di cui il Prof. Camillo Benso Ballabio (risale a quell'epoca la metamorfosi del nome, con il quale in seguito divenne noto) venne nominato dirigente. Il Centro oltre all' attività ambulatoriale aveva la disponibilità di una sezione di 16 letti nell' ambito della Clinica Medica. Nel 1962 Ballabio veniva nominato "Professore incaricato di Reumatologia" presso l'Università di Milano.

Un primo periodo dell'attività scientifica del Ballabio, dal '36 al '45, comprende numerosi contributi su diversi argomenti di Clinica Medica Generale, sicché nella lettera di presentazione del suo Maestro alla Libera Docenza del 1951 è detto come Ballabio abbia portato "contributi a svariati argomenti clinici, biochimici, semeiologici, ematologici e terapeutici". In particolare studiò un tipo di infezione tubercolare attenuata, con spunti per problemi di patogenesi tubercolare e di immunità antitubercolare. Alla luce delle attuali conoscenze tali indagini acquistano particolare interesse, per aver dimostrato Ballabio, tra i primi, la possibilità di trasferire passivamente uno stato di ipersensibilizzazione a mezzo di estratti d'organo, specie se ricchi in linfociti. Queste ricerche in veste monografica gli valsero il conseguimento del premio $\mathrm{Ga}-$ nassini 1949.

Col precisarsi del suo indirizzo di studi in campo reumatologico, Ballabio condusse estese ricerche sulle proprietà terapeutiche di alcuni steroidi nei confronti di alcune malattie reumatiche, giungendo all' affermazione che nell' ambito di queste sostanze dovessero riconoscersi farmaci attivi in senso antireumatico. Con la scoperta del cortisone, di cui fu tra i primissimi studiosi, Ballabio indirizzò le sue ricerche su questo steroide e sui suoi derivati. I suoi studi sul composto $\mathrm{E}$ od $\mathrm{F}$ sono tra i più estesi e documentati della letteratura europea e culminarono nella monografia "Il cortisone, reu- matismo e problemi affini" premiata in pubblico concorso nel 1951 e nella relazione al VI Congresso Italiano di Reumatologia, tenutosi a Taormina nel 1952. Sempre nel 1952 Ballabio assieme al suo Maestro organizzava a Milano un "Symposium Europeo sul Cortisone", cui partecipava con una relazione sul trattamento cortisonico delle carditi evolutive. Sempre in questo filone di ricerche, Ballabio sperimentò l'impiego dei derivati cortisonici nei versamenti pleurici e pericardici, conseguendo per questi studi il Premio Marzotto per la Medicina 1954 con l'opera "L'acetato di idro-cortisone nel trattamento locale delle malattie reumatiche" in collaborazione con G. Sala, A. Amira e L.P. Minetti. Ballabio inoltre fu il primo ad occuparsi nel mondo dei Delta1-derivati, di cui dettò tutte le principali proprietà, nonché dei fluoro-derivati.

Le ricerche scientifiche di Ballabio toccarono in seguito numerosi altri capitoli della reumatologia. Spiccarono tra le altre quelle sulla gotta, con le quali, in collaborazione con gli allievi della Scuola del Prof. Villa, potè dimostrare come l'acido urico sia presente nel sangue in forma libera e quindi interamente filtrabile. Nello stesso settore di ricerche egli dimostrò, sempre in collaborazione con la scuola reumatologica milanese, l'importanza della escrezione renale dell'acido urico nei confronti dell'aumentato "pool" d'acido urico, fattore patogenetico oggi universalmente riconosciuto. Questi studi culminarono nella relazione al Congresso Internazionale di Reumatologia di Toronto, in collaborazione col Prof. Villa e col Prof. Robecchi e con la scuola reumatologica francese del Prof. De Sèze (1958).

Nei confronti dell'artrite reumatoide Ballabio, oltre ai citati ampi contributi in tema di terapia cortisonica, condusse studi approfonditi sulla sua variante maligna e sui rapporti di quest'ultima con il quadro che descrisse e omologò come "collagenopatia cortisonica". Fu tra i primissimi, mediante indagini all'ultracentrifuga condotte in collaborazione con la sua allieva Elisa Cirla, a dimostrare la presenza di fattori reumatoidi in eccesso nel sangue di questi soggetti sotto forma di complessi $22 \mathrm{~S}$ e $10 \mathrm{~S}$; così come fu tra i primi, utilizzando la metodica dell'immunofluorescenza diretta da poco introdotta in campo medico, in collaborazione con Benvenuto Pernis, a dimostrare la presenza di fattori reumatoidi nei vasi e nei tessuti. Per questo gruppo di studi ebbe citazione di merito al Premio San Remo di Reumatologia, assegnato alla Prof. Nanna Svartz di Stoccolma. 
Nel campo del reumatismo articolare acuto Ballabio, in collaborazione con il suo Maestro Villa e con G. Sala, si occupò intensivamente di patonomastica, di patogenesi e di terapia, precisando quella che ancor oggi è comunemente riconosciuta come la "quarta fase o fase di cronica attività della malattia reumatica". Su alcuni aspetti della cardiopatia reumatica Ballabio con Bregani e Ortenzi fu relatore al XII Congresso della Società Italiana di Reumatologia a Bologna nel 1958. Le sistematiche ricerche epidemiologiche condotte da Ballabio sulla malattia reumatica in collaborazione anche con la Clinica Pediatrica di Milano culminarono nella relazione al II Congresso Internazionale di Medicina Profilattica di Merano del 1952, nella relazione di Scuola al XIV Congresso della Società Italiana di Reumatologia a Bologna nel 1961 e nella relazione col suo Maestro Villa al XXIII Congresso della Società Italiana di Cardiologia del 1962.

Ballabio si occupò ripetutamente anche di problemi patogenetici e nosografici in tema di artrosi. Tra i primissimi puntualizzò il concetto di "artrosi primaria", oggi universalmente accettato.

Nel settore terapeutico, oltre ai già citati lavori in materia di terapia cortisonica, Ballabio condusse ricerche si può dire nei confronti di tutti i principali medicamenti antireumatici della sua epoca. Particolare risalto ebbero le sue ricerche sul fenilbutazone e i suoi derivati, preconizzando l'uso endovenoso della butazolidina, e sull'attività urico-eliminatrice del sulfinpirazone. Queste ricerche costituirono il tema della relazione tenuta con il suo Maestro Villa al VI Congresso Internazionale di Terapia di Strasburgo nel 1959. Si è detto come Ballabio sia stato il primo ad introdurre in Europa il trattamento dell'artrite reumatoide con antimalarici di sintesi; ricerche sistematiche fin dall'inizio condusse inoltre sulla terapia dell'artrite reumatoide con i sali d'oro, in rapporto ai singoli preparati ed alla associazione con i cortisonici. Importanti furono pure i suoi contributi sull'uso terapeutico dell' indometacina e degli altri farmaci antireumatici non steroidei di seconda generazione. Fu tra i primissimi a preconizzare l'impiego delle mostarde azotate nell' artrite reumatoide.

Nel 1965 veniva data alle stampe la traduzione italiana del Trattato di Reumatologia di J.L. Hollander, ampiamente commentato e integrato da Ballabio, che lo arricchì con la rassegna critica dei contributi originali in materia delle diverse scuole reumatologiche italiane, opera in due volumi che doveva diventare l'abbecedario di ogni reumatologo italiano della "seconda generazione".

Il $1^{\circ}$ agosto 1967 iniziava un nuovo corso nella vita del Prof. Ballabio: veniva inaugurata presso l'Istituto Ortopedico Gaetano Pini la Divisione di Reumatologia, di cui egli aveva vinto il concorso per il primariato: la prima e per molti anni l'unica Divisione di Reumatologia della Lombardia. L'organico era costituito, oltre che dallo stesso Prof. Ballabio, dal vincitore del concorso per un aiuto ospedaliero, il Prof. Alfonso Amira, e dai vincitori del concorso per tre posti di assistente ospedaliero, la Prof.ssa Elisa Cirla (che diverrà successivamente Primario del Laboratorio dello stesso Istituto), il Dott. Innocenzo Caruso e il Dott. Flavio Fantini. Integrava il gruppo il Prof. Bruno Colombo in qualità di Assistente Universitario. Trasferendosi dal Padiglione Granelli del Policlinico all'Istituto Ortopedico Gaetano Pini, Ballabio portava con sè altri numerosi collaboratori, alcuni dei quali verranno col tempo inseriti nell'organico dell'Istituto, come la Dott.ssa Giuliana Gilardi (divenuta in seguito Primario del Servizio di Cardiologia dello stesso Istituto), il Dott. Glauco CheriéLignière, il Dott. Sergio Tosi, il Dott. Mario Carrabba. L'inserimento del gruppo reumatologico nella gloriosa istituzione milanese, fondata nel 1874 come "Pio Istituto dei Rachitici" e divenuta Clinica Ortopedica dell'Università nel 1924, apriva tutto un nuovo panorama di esperienze: lo stretto rapporto di collaborazione con gli ortopedici, l'attiva disponibilità della Sezione Ricerche dell'Istituto propugnata dall'allora Direttore Sanitario dell' Istituto, Prof. Gianfranco Acchiappati, l'ampliamento dello staff permettevano in pochi anni di approfondire studi e ricerche in svariati campi della reumatologia, sia "di base" (artrosi sperimentale; aspetti immunitari della cardite reumatica, dell'artrite reumatoide e del lupus; istologia della sinoviale; istochimica della cartilagine), sia clinici (artropatie microcristalline, polimialgia reumatica, reumatismi cronici dell'infanzia), sia terapeutici (effetti secondari dei cortisonici, sinoviectomia, sinoviortesi, immunosoppressione, nuovi farmaci antiinfiammatori non steroidei). Corollari dell'attività scientifica svolta in seno all' Istituto Gaetano Pini dovevano essere l'istituzione nello stesso anno 1967 delle Giornate OrtopedicoReumatologiche omonime e la costituzione in data $1^{\circ}$ novembre 1978 dell'Istituto Policattedra di Patologia e Clinica dell' Apparato Locomotore, realizzato con il Prof. Luigi Parrini, Ordinario di Clinica Ortopedica, che ne fu il primo Direttore. Nel 1961 Ballabio aveva partecipato all'organiz- 
zazione del X Congresso della Lega Internazionale contro il Reumatismo (ILAR), che si svolse a Roma (per l'unica volta in Italia), in qualità di segretario generale. Dal 1965 al 1969 Ballabio fu Assistant President della Lega Internazionale contro il Reumatismo a fianco di Alessandro Robecchi, suo carissimo e indimenticato amico, di cui, assieme al devoto allievo Vittorio Daneo, portò a termine la stesura e curò la pubblicazione del monumentale "Trattato di Reumatologia" (1971), dopo la prematura scomparsa del grande reumatologo torinese.

Nel 1971 in seguito a regolare concorso Ballabio veniva nominato Professore Straordinario di Reumatologia dell'Università di Milano, nel 1974 gli veniva conferito l' ordinariato. A conferma dell'alta considerazione di cui godeva nel mondo medico-scientifico nazionale, nel 1975 gli venne affidato il compito dalla Società Italiana di Medicina Interna di tenere la relazione magistrale al 76mo Congresso della Società. Il 28 settembre di quell'anno, a Riva del Garda, Ballabio affrontò e svolse con la consueta competenza e vivacità espositiva il tema su "La Polimialgia Reumatica e la Malattia di Horton". La relazione suscitò grandissimo interesse ed ebbe il merito di far conoscere una patologia allora ancora poco nota al di fuori della stretta cerchia degli addetti ai lavori e che doveva diventare, anche in rapporto con il progressivo aumento della speranza di vita della popolazione, una delle forme reumatiche epidemiologicamente più rilevanti.

Nel 1972 frattanto Ballabio era riuscito a istituire a Milano, tra le prime in Italia, la Scuola di Specializzazione in Reumatologia. I primi diplomati (4 luglio 1975) furono (in ordine alfabetico): Alfonso Amira, Mario Carrabba, Innocenzo Caruso, Glauco Cherié-Lignière, Bruno Colombo, Enzo D'Ingianna, Flavio Fantini e Sergio Tosi. Frequentarono la Scuola di Milano e si specializzarono in Reumatologia sotto la direzione di Ballabio tra i tanti anche Bianca Marasini e Silvano Todesco (1977), Leonardo Punzi (1978), Bianca Canesi e Vittorio Modena (1979), Luigi Frizziero (1980), Gianfranco Ferraccioli (1981).

Nel triennio 1973-75 Ballabio svolse il compito di Presidente della Società Italiana di Reumatologia e dal 1976 ricoprì l'incarico di Presidente della della Lega Italiana contro il Reumatismo e per l'aiuto al malato reumatico (LIMAR).

In aggiunta ai suoi grandi meriti scientifici e alle straordinarie capacità organizzative, non va dimenticato che Ballabio è stato anche un medico insigne. La Medicina è allo stesso tempo Scienza ed Arte e quindi appaga la sete di conoscenza in termini positivi, ma anche lascia spazio all'intuito e alla creatività. La Medicina è inoltre contatto con l'Umano, con il soma ma anche con la psiche, e pertanto era congeniale a chi, come Ballabio, aveva una istintiva curiosità dell'uomo e delle sue passioni e una innata facilità nel rapporto interpersonale.

Ballabio era insuperabile nel raccogliere il sintomo decisivo per la diagnosi, che si trattasse di un soffio diastolico, di uno sfregamento pleurico o di una "milzetta". Al letto del malato per chi lo seguiva nella visita era un continuo esame di clinica medica. Pur essendo un virtuoso del ragionamento clinico, era un convinto assertore del "nozionismo medico": non poteva ammettere che praticasse la medicina chi non sapesse enumerare senza indugi gli 8 caratteri del polso (frequenza, forza, ampiezza, tensione, durezza, rapidità, ritmo, uguaglianza) o i 7 caratteri di una massa palpabile (grandezza, forma, delimitazione, consistenza, aderenza, dolorabilità, fluttuazione). Seguace del grande Murri nell'affermare che "per fare diagnosi di una malattia, bisogna prima pensarla", era un accanito classificatore degli stati morbosi. Questa sua passione per le classificazioni delle malattie poteva sembrare vuoto accademismo solo a chi non comprendeva la funzione euristica che la classificazione doveva avere, guidando il medico nella diagnosi attraverso un procedimento di confronto del singolo caso clinico con tutti i possibili stati morbosi. Nell'introduzione al suo lavoro sulla Classificazione delle Malattie Reumatiche pubblicato sulla prestigiosa rivista Recenti Progressi in Medicina (1971), scriveva dunque Ballabio: "Classificare è una esigenza dell'intelligenza e del conoscere umano attraverso il dato dell' esperienza che ci addita, in una serie di oggetti o di fatti, le analogie e le differenze, donde la possibilità di derivarne i caratteri principali e invariabili, accanto a quelli accessori o contingenti. Ne consegue una disposizione ordinata che la logica percorre, ai fini di una identificazione e di una rievocazione le più rapide ed esatte possibili." E aggiunge: "Lo studioso che si accinge a così ardui e delicati compiti deve possedere una mentalità aperta alla diversità dei pareri, aliena da personalismi aprioristici e porsi come pregiudiziale il tipo di classificazione che intende attuare: qualunque essa sia, eziologica, didattica, anatomica, una classificazione deve possedere una logica interna, una patonomastica adeguata a ciò che si vuole esprimere, ricordando di collocare le 
parole sui concetti e non viceversa." Dopo la minuziosa esposizione della sua classificazione l' $\mathrm{Au}$ tore concludeva: "La classificazione che abbiamo compilato ha, come ogni classificazione, un valore pragmatico ed è pertanto aperta ad ogni critica, che non mancherà e che desideriamo; l'argomento, come tale, è in continua trasformazione, soggetto come è al progredire delle nostre conoscenze e non può mai considerarsi esaurito; non saremo certo noi ad aver detto l'ultima parola in argomento.

Ma proprio per il fine che ci siamo proposti, quello cioè di realizzare una classificazione clinica la più aggiornata possibile, non crediamo di aver fatto cosa superflua, offrendo al medico la possibilità di richiamare alla sua mente nel modo più pronto e preciso possibile una diagnosi esatta, condizione indispensabile per una prognosi e una terapia adeguate."

Le malattie reumatiche, quale argomento di lezione o di conferenze, non hanno certo il fascino delle malattie del sangue o delle ghiandole endocrine, oppure il sinistro richiamo delle coronarie o del cancro, quindi è difficile credere all' attenzione ammirata e all'entusiastica accoglienza ogni volta riservate alle parole di Ballabio, quando il Maestro in una delle tante serate si rivolgeva ai medici di Sondrio, o di Bergamo, o di Varese, o di Vaprio. Ballabio sapeva trasformare ogni argomento in una vivida sequenza di immagini, ora drammatiche, ora curiose, talora anche umoristiche, sempre interessanti; con ritmo incalzante le sue parole commentavano le diapositive, che ogni volta con immensa cura sceglieva tra le sue molte o appositamente preparava. Ma il clou della serata era di solito rappresentato dal momento nel quale si apriva la discussione: se i colleghi, provati dalla precedente martellante esposizione, o timorosi di esporsi, non erano solleciti nel formulare qualche quesito, incredulo Ballabio si guardava intorno ed esordiva "Eppure su questo argomento si potrebbe fare tutta una serie di domande; se nessuno ne propone, allora mi rivolgerò io stesso una domanda..." e riprendeva con foga inesauribile.

Pochi docenti, come Ballabio, amarono così profondamente gli studenti. Forse perché privato della gioia di avere dei figli suoi, investiva nei suoi allievi una grande parte del suo patrimonio affettivo. Con ogni mezzo si adoperava per accendere in loro il desiderio di conoscenza e trasmettere loro il senso di responsabilità che la condizione di medico comporta, e perché i futuri medici avessero della Medicina non una gretta concezione strumenta- le, ma ne facessero veicolo di Umanità.

Nel luglio del 1976 Ballabio, nel pieno della sua maturità fisica ed intellettuale, veniva colpito per la prima volta dal terribile male che non gli avrebbe dato tregua. Con grande coraggio, con volontà di ferro, con pazienza infinita si sottopose a tutte quelle sfibranti procedure che dovevano di nuovo insegnargli a camminare, a controllare i movimenti della mano, ad articolare correttamente la parola. Nel giro di pochi mesi, nell'incredulità generale, ritornò completamente padrone di se stesso; a poco a poco la sua vita riprese il ritmo frenetico di prima. Ma se, come spesso citava "valere est, non vivere vita", l'uomo non poteva più essere quello di prima: giorno e notte lo accompagnava un indicibile dolore, una causalgia refrattaria ad ogni trattamento.

Nell'agosto del 1981 venne, implacabile, la recidiva. La vita del Maestro divenne uno straziante ed umiliante Calvario. Il 20 aprile 1984, Venerdì Santo, veniva infine la morte, tanto invocata ma anche tanto temuta.

È opportuno a questo punto citare la testimonianza del Prof. Bruno Colombo, uno dei più cari allievi di Ballabio, accanto al quale aveva trascorso una intera vita e che gli succedette nella posizione di Direttore della Cattedra di Reumatologia e di Primario della Divisione di Reumatologia del Gaetano Pini. Scriveva dunque Colombo del suo Maestro nel Numero Speciale di Reumatismo (1989): "Fu un uomo di profonda cultura umanistica, capace di affrontare argomenti anche in parte estranei alla specialità ed alla stessa Medicina; ne è un esempio il suo saggio: "Il dolore cronico nella Società" sugli aspetti fisici, psicologici ed esistenziali del dolore, scritto nel 1979 quando già da tre anni era ammalato ed accusava una tormentosa sintomatologia a tipo parestesia dolorosa che lo rendeva spesso nervoso e per la quale chiedeva a noi suoi allievi i mezzi per combatterla; quindi saggio scritto da persona che provava egli stesso il dolore. Altri saggi che denotano la sua vastità di cultura e la sua genuina origine milanese di cui si vantava sono: "La malattia di S. Ambrogio e in S. Ambrogio" e "Il Porta nella medicina e la medicina nel Porta".

Dalla sua affascinante conversazione trasparivano spesso i dubbi esistenziali che lo tormentavano e dei quali cercava la soluzione nelle letture dei classici latini, della Divina Commedia, degli arrabbiati francesi quali Baudelaire, di D'Annunzio fino all'ermetico Ungaretti, all'angoscioso Montale e, in consonanza con la sua ipersensibilità, a Proust. 
Riteniamo cosa utile concludere questo scritto con alcuni brani del Maestro, che, in quanto non relativi a materia medica e quindi facilmente databili, ma relativi all'approccio al malato cronico, restano di grande attualità e costituiscono un perenne insegnamento. Scrive Ballabio nel suo saggio su S. Ambrogio: "Chi scrive è reumatologo e ha molta familiarità con pazienti affetti da malattie inguaribili, iperalgiche; sulla scorta di esperienza ormai annosa non esito ad affermare che è proprio in questi pazienti che noi vediamo come ogni malato ha la "sua" malattia, non tanto e non solo in chiave organica, ma anche e soprattutto nei suoi riflessi psicologici.

È delle malattie croniche e debilitanti quel tipo di sofferenza che fa dire a Milton nel "Lost Paradise": "Pain is perfect misery, the worst of all evils; and excessive overturns all patience", in presenza di un avvenire che non è tanto quello cui essi vanno incontro quanto quello che viene loro incontro; sotto il peso come sono del loro "handicap", dello stato di dipendenza fisica ed economica in cui vengono a trovarsi, questi pazienti virano verso comportamenti che si centrano su poche possibilità: $o$ rinunciare ad ogni opzione positiva fino alla disgregazione del loro io fisico ed intellettuale (sono quelli che Engel definisce "Pain prone patients") o adottare la "carriera di malati" attuando una strategia di sfruttamento delle loro sofferenze favoriti in questo da situazioni iperprotezionistiche familiari o dai mezzi assicurativi di cui possono beneficiare, o infine virare verso posizioni di rancore, di invidia e di protesta tanto più gravi quanto meno noi forniremo loro una giustificazione del loro stato, un significato alla loro esistenza, un reinserimento nella società.

Sono questi i pazienti che sono messi a più dura prova nel trovare un significato alla malattia e che più facilmente ne vengono condizionati, fino alla conversione nevrotica; sono questi i pazienti che più degli altri hanno bisogno di un aiuto, per interpretare il proprio destino, colpiti gravemente come sono nelle loro relazioni familiari e sociali.

Ad essi conviene un approccio tutto particolare, approccio che deve tener conto dell' ammalato nella sua singolarità, nella sua rete interrelazionale di affetti, nel suo ruolo professionale.

E sono questi purtroppo i pazienti che il medico rifiuta più facilmente, brutalizzandoli talora con terminologie traumatizzanti quali quelle di deformante, di cronico, di progressivo, capaci di psichiatrizzarli nel modo più pericoloso.

Il medico, messo continuamente in iscacco dall' in- successo delle cure, prova la sensazione di una irritata impotenza, che lo pone di fronte alla frustrazione di quella infantile illusione che aveva spesso determinato la scelta della sua carriera: quella di poter guarire tutto e tutti!

$\mathrm{Ne}$ consegue un rifiuto che altro non è se non la fuga messa in atto dalla stessa angoscia del curante, che finisce per condizionare un ciclo vizioso.

Solo offrendo a questi malati un altro modo di vivere noi li aiuteremo a uscire da posizioni rinunciatarie, solo indirizzando adeguatamente la loro concettualizzazione del dolore, solo ridando fiducia al loro "sviluppo vitale" noi li abitueremo a superare il loro "handicap" così come insegna Ambrogio: "Non enim in passione esse, sed victorem esse passionis beatum est, nec frangi temporalis motu doloris" (Off. II, 5, 19) (Non l'adagiarsi nella sofferenza, bensì il saperla vincere è cosa beata, né ci si deve lasciare distruggere dalla violenza del dolore fisico).

Per questo la società e ciascun componente di essa hanno il dovere di avvicinarsi alla sofferenza; aiutando questi pazienti a sopportare la loro minorazione fisica, che vuol dire anche ridurne le gravi ripercussioni sul morale; ciò è tanto più valido per il malato cronico, così spesso trascurato là dove maggiore è la necessità che il paziente non si lasci sovvertire dal dolore, ma affronti il suo handicap come un evento avverso tra gli altri e i tanti della vita. Ciò lo aiuterà a polarizzare la sua volontà verso il superamento del suo handicap, utilizzando quell' ancor molto del suo Io che gli permetterà di ritrovare nella sua esistenza un significato e uno scopo, che coincidano colla sua volontà."

Il 6 dicembre 1980, in occasione del I Congresso della Lega Italiana contro il Reumatismo e per l'Aiuto al Malato Reumatico, tenutosi a Milano nell'Aula Magna dell'Istituto Ortopedico Gaetano Pini, alla fine della sua prolusione intitolata "La mano e l'uomo" Ballabio concludeva con queste parole: "E sia compito di questo nostro incontro, tra Autorità, medici, pubblico e malati quello di "dar loro una mano" che li aiuti a ritrovare, attraverso la massima reintegrazione delle loro capacità fisiche e funzionali, la fiducia in un reinserimento attivo nella società, che restituisca loro fiducia in uno "sviluppo vitale" sempre più elevato, unico e vero motivo dell'esistenza, come passaggio dalla potenza all'atto. Passaggio che Michelangelo vide attuarsi attraverso il contatto, quanto mai indicativo, del dito di Dio con quello dell'uomo.

E mi sia concesso consegnare a voi tutti una con- 
vinzione emersa dal mio lungo esercizio professionale, quella che se anche poco riusciamo a dare a questi ammalati, la loro gratitudine sarà am- piamente rimunerativa della pazienza, tenacia e generosità con cui ci saremo dedicati al difficile compito."

\section{RIASSUNTO}

In questo scritto l'autore ricorda, a vent' anni dalla morte, Camillo Benso Ballabio, indimenticabile maestro di reumatologia e di medicina, clinico insigne e uomo di profondissima cultura. Vengono ripercorse le tappe più importanti della sua vita, integrando le note biografiche con alcune citazioni che testimoniano lo spessore intellettuale di questo protagonista della storia della reumatologia italiana.

Parole chiave - Storia della reumatologia - Italia.

Key words - History of rheumatology - Italy. 